\title{
Impact of Mobility on Last Encounter Routing Protocols
}

\author{
Fan Bai*, Ahmed Helmy* \\ ${ }^{*}$ General Motors Research Center, General Motors Corporation \\ ${ }^{\dagger}$ Computer and Information Science and Engineering Department, University of Florida
}

\begin{abstract}
In this study, we analyze last encounter based routing protocol (e.g., FResher Encounter SearcH, or FRESH) that utilizes encounter history to create time (or age) gradients for information diffusion in wireless networks. FRESH protocols can be used for resource discovery, routing or node location, and hold great promise for future wireless networks.

We provide the first study on sensitivity of this class of protocols to a rich set of mobility models (Manhattan, Group, Random Walk and Random Waypoint models). We find that FRESH is sensitive to the mobility pattern. However, somewhat to our surprise, FRESH's performance after warm-up is insensitive to velocity for all the mobility models examined. To expose the fundamental reason behind these observations, we develop analytical models to analyze FRESH's performance and validate these models via extensive simulations. Finally, our analysis concludes that the characteristics of the age gradient tree is the key factor to explain this interplay between mobility and the performance of FRESH protocols.
\end{abstract}

\section{INTRODUCTION}

Recent studies [6] [7] indicate that mobility imposes negative effects on conventional routing protocols (e.g., DSR, AODV, DSDV, etc.). However, mobility also provides opportunities that can, and in fact should, be utilized to enhance the performance of MANET protocols. For example, last encounter based protocols (e.g., EASE [5], or FRESH [4]) explicitly use the node mobility and encounter history to locate (and establish routes to) other nodes. In this paper, we focus on FRESH protocol [4], as a generic example for this type of last encounter based protocols. FRESH uses node encounter history to create time (encounter age) gradient tree within the network. By following the time (or age) gradient, a mobile user can efficiently establish routes to, or locate, other users. The age gradients are created according to the node encounter patterns, which in turn depend on the node mobility patterns. However, this complicated interplay between mobility and mobility-assisted last encounter based protocol had not been investigated and analyzed yet. We present the first such study in this paper, in order to fill the important gap in the quantitative understanding of mobility-assisted protocols and their interaction with mobility process, helping to design and further improve the mobility-assisted protocols.

In this paper, we aim to (1) develop a deep insight into the protocol mechanisms for the last encounter routing protocols (e.g., FRESH) and (2) gain a better understanding of the interaction between the FRESH protocols and the mobility process. Particularly, we are interested in answering the questions: How does the performance of the FRESH protocols vary with different mobility patterns and velocity? and Why?

We use both extensive simulations and theoretical analysis to answer these questions. First, we conduct extensive simulations using a rich set of mobility models, including Manhattan, Group mobility, Random walk and Random waypoint models. Through simulations, we find that the warm-up behavior is highly sensitive to both the mobility model and node velocity. We also confirm that the steady state behavior is only affected by the different mobility patterns but is insensitive to the node velocity. Then, to explain these interesting observations, we develop a set of analytical models for both warmup behavior and steady behavior of FRESH protocol. These analytical models, together with the simulation results, provide initial insight into the performance trend of the FRESH mechanisms. The analytical solutions we provide are not specific to a particular mobility model.

To develop a clear picture about the effect of mobility on FRESH protocol, we identify a key characteristic called the temporal-spatio correlation that refers to the property that the nodes having 'fresher' encounter ages are closer to the destination. Through simulation, we observe that temporal-spatio correlation exists in all the mobility models we studied. We use temporal-spatio correlation and its derivant, Age Gradient Tree (AGT), 
to explain the insensitivity of FRESH to velocity for the various mobility models. Our analysis, findings and insights pave the road for further research in the area of last encounter routing protocol, which is fundamentally different from traditional protocols. The contributions of this study include:

1) We provide the first sensitivity study for the FRESH protocols: The transitional behavior of FRESH protocol is sensitive to both mobility pattern and velocity. We also find that its steady state behavior is insensitive to velocity, but still sensitive to the mobility pattern.

2) We develop novel analytical models to capture performance of FRESH in both phases and validate our models through extensive simulations, over a rich set of mobility models.

3) We introduce two new metrics - temporal-spatial correlation and characteristics of age gradient tree - as key part of our analysis to understand the complicated interplay between mobility and FRESH.

\section{RELATED WORK}

Ref. [2], which showed that node mobility can be utilized to dramatically improve network capacity, was the first work to point out that mobility can be a positive factor. A large number of works were then proposed to overcome network partition or facilitate packet delivery by utilizing node mobility, such as SWIM [3], DTN [1], Message Ferry [8]. Beside them, EASE [5] and FRESH [4] introduce a revolutionary paradigm for routing protocols, which explicitly use last encounter information to successively refine the estimation of network topology and deliver the packets. EASE is a geographic location discovery scheme that solely relies on information about node encounter history (both the time and location of node encounters) [5]. Even if the geographic location of node encounters is unknown, the last encounter still could be used to improve protocol performance (i.e., reduce the search overhead), as shown in FRESH [4].

Inspired by these two studies and to further explore this research topic, our study mainly concentrates on filling the gap of analyzing the complicated interaction between various mobility patterns and encounter-based FRESH protocol. To do so, beside the Random Walk and Random Waypoint models in [4] [5], we evaluate the FRESH protocol performance across an even richer set of mobility models. Moreover, our analytical models are appropriate for all these mobility models, rather than only focusing on the asymptotic performance in the
Random Walk model [5]. Finally, beyond the previous works [4] [5], we explore the fundamental design principle behind FRESH protocol and then identify that the temporal-spatial correlation is the key to explain the complicated interaction between mobility and FRESH protocol.

\section{LASt EnCounter BASEd Routing Protocol}

Different with conventional MANET routing protocols that purely rely on spatial information of location to find the route, the establishment of routes in the FRESH protocols is done by the guidance of temporal information of node encounter. The intuition behind FRESH protocol is to utilize the so-called temporal-spatio correlation of the existing mobility models, i.e., the Cartesian distance (spatial information) between two nodes is more or less correlated with the time when they encounter with each other (temporal information).

\section{A. Mechanisms of FRESH Protocol}

Thus, the mechanisms ${ }^{1}$ of FRESH protocols are centered on how to record and how to utilize the node encounter history, including two phases:

Encounter caching phase: As two nodes move within the transmission range, both nodes record the time and location of encounter and the $\mathrm{D}$ of the other. Initially, each node only caches the nodes within its direct neighborhood(cold-cache phase). Later, after a node has encountered a large portion of the other nodes, it is able to develop a richer and more accurate view of the whole network topology(warm-cache phase). Clearly, sufficient encounter events (and, hence time) are needed to populate (warm up) the encounter tables, so that FRESH protocol can gradually change from the 'coldcache' phase to the 'warm-cache' phase. We call this time of cache state transition as the warm-up time.

Route Searching Phase: Each node is able to utilize node encounter history to discover the destination node, by iteratively finding a series of intermediate nodes which had encountered the destination with the decreasing encounter age $^{2}$ for the given destination. In each step, the intermediate node searches for the

\footnotetext{
${ }^{1}$ The FRESH protocols could have other variants, in terms of detailed mechanisms. In this paper, the design choices of studied FRESH protocol are specified as Ref. [4]: Only time of encounter is recorded; fixed-incremental expanding ring search; and greedy search strategy. However, the conclusions and methodologies presented in this paper also apply to the other variants.

${ }^{2}$ Encounter age $(A G E)$ is the difference of the current time and the node encounter time, and it indicates the 'freshness' of node encounters.
} 


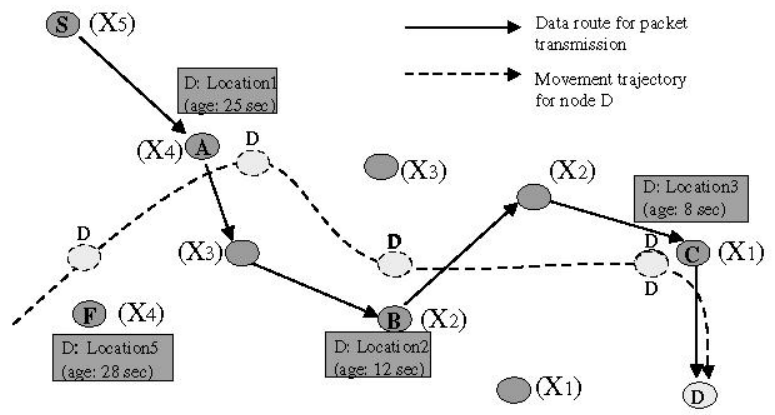

Fig. 1. The Example of FRESH Protocol Operation. In this example, to better illustrate how FRESH protocol works, we assume that all the other nodes except node D are stationary. Here, the $\left(X_{n}\right)$ symbol indicates the hop length of this node to destination, in terms of SPT distance. For example, via SPT tree, node $\mathrm{C}$ is 1-hop from destination $\mathrm{D}$, and node $\mathrm{B}$ is 2-hops from destination.

next intermediate node that encountered the destination even more recently. This way, the search procedure is repeated until it finally reaches at the destination. In each search step, an anchor with a larger encounter age is called upstream anchor and an anchor with a smaller encounter age is called downstream anchor. The search is conducted according to the search method, in a manner similar to the expanding ring search.

As illustrated in Fig.1, the source $S$ utilizes the FRESH protocol to discover the destination $D$. Node $S$ first searches in its neighborhood and discovers node $A$ (that encountered $D 25 \mathrm{sec}$ ago) as its downstream anchor, and node $A$ then finds node $B$ (that encountered $D 12 \mathrm{sec}$ ago). Node $B$, in turn, finds node $C$ (that encountered $D 8 \mathrm{sec}$ ago) and finally node $C$ directly locates node $D$ in its neighborhood.

\section{B. Age Gradient Tree for FRESH Protocol}

In FRESH protocol, each upstream anchor is responsible for discovering its downstream anchor. This way, source node eventually finds the destination via a series of concatenated links, with each link pointing from an upstream anchor with larger encounter age to a downstream anchor with smaller encounter age. This set of concatenated links forms an age gradient path with a strictly decreasing encounter ages, from the source to the destination. For a given destination, the collection of age gradient paths from various source forms an Age Gradient Tree(AGT) rooted at this destination. Clearly, the data packets in FRESH protocol are sent via age gradient tree. In contrast, in the conventional routing protocols, the packets are sent to destination via the Shortest Path Tree(SPT) (Generally, Age Gradient Tree is different from Shortest Path Tree). As shown later, we identify that the characteristics of age gradient tree are the key element to study the interaction between mobility and FRESH protocol performance.

\section{Simulation Settings}

\section{A. Mobility Models}

To thoroughly examine the performance of FRESH protocol, we evaluate the FRESH protocol over a rich set of mobility models. These models are carefully chosen so that each of them exhibits different mobility characteristics:

(1) Random WayPoint (RWP) model: Each mobile node randomly selects one location and moves towards it with a randomly chosen speed. Upon reaching the destination, node stops for a certain period and then moves towards another randomly chosen destination. In RWP model, the node velocity is independent of other nodes. However, for a given node, the current node velocity depends on its previous one. Hence, RWP exhibits a strong degree of temporal correlation and weak degree of spatial correlation.

(2) Random Walk (RWK) model: In RWK model, the nodes change their speed $V(t)$ and direction $\theta(t)$ at each time interval $t$. Both RWK and RWP model exhibit strong randomness, while RWK model exhibits a weak degree of temporal correlation.

(3) Reference Point Group Mobility (RPGM) model: RPGM model is used to model group mobility. Here, each group has a group leader and a number of group members, with each group member choosing a velocity by randomly deviating from its group leader. Thus, the node movement in RPGM model is correlated with its groupmates. The RPGM model is expected to show a strong degree of spatial correlation between different nodes.

(4) Manhattan (MH) model: MH model emulates the node movement on streets. Manhattan grid maps of horizontal and vertical streets are used to restrict the node movement. On each street, the mobile nodes move along the lanes of both directions with randomly chosen speed. Unlike RWP model, the mobile nodes only travel on the pathways in the map.

These models represent a rich set of mobility characteristics varying from weak to strong degrees of temporal correlation, spatial correlation and geographic restriction, providing a solid basis to evaluate and analyze the FRESH protocol.

\section{B. Simulation Setting}

We carry out the simulation in our customized event-driven simulator. The mobility traces are obtained 
through the IMPORTANT mobility scenario generator [7], by following RWP, RWK, RPGM and $\mathrm{MH}$ models. In all these patterns, 400 mobile nodes move in an area of $3000 \mathrm{~m}$ by $3000 \mathrm{~m}$ for a period of 4000 seconds. The value for the radius range is set as the default value $(250 \mathrm{~m})$. For RPGM, 80 groups (with five nodes for each group) are moving independent of each other. The maximum speed is set to $5,10,20,30,40$ and $50 \mathrm{~m} / \mathrm{s}$ to generate different movement patterns for the same mobility model. To minimize the potential impact of data traffic patterns on protocol performance, we use a simple pattern consisting of 100 pairs of CBR traffic with randomly chosen source and destination. The data rate used is 4 packet/sec. We used different random seeds to generate five different traffic patterns for each mobility scenario.

\section{Performance Evaluation of Fresh PROTOCOL}

Extensive simulations were conducted in this section to answer the question How FRESH protocol mechanisms and their performance are affected by the mobility scenarios?

\section{A. Transitional Behavior and Steady Behavior}

We are particularly interested in examining two performance metrics, overall average path length $\overline{P L}$ and overall average search cost $\overline{S C}$, to compare FRESH protocol performance across various mobility models. $\overline{P L}$ (or, $\overline{S C}$ ) is the average path length of route (or, the average number of search packets) generated by the FRESH route.

We record the values of $\overline{P L}$ and $\overline{S C}$ at each time instance. Interestingly, it is clearly found that the FRESH protocol performance cannot be stabilized at the initial phase. For example, in RWK model, $\bar{P} L$ constantly keeps increasing (as shown in Fig.2(a)) and $\overline{S C}$ keeps decreasing (as shown in Fig.2(b)) as time elapses. Both of them become relatively stable after some time (around 545 seconds) and reach a steady state. Thus, the operation of the FRESH protocol can be divided into two states: initial transitional state and follow-up steady state. Similar phenomenon are also observed for RWP, RPGM and MH models.

This phenomenon is caused by the different caching phases of the FRESH protocol. At the cold-cache phase, FRESH protocol mostly relies on the flooding mechanism to search the route, since it is difficult to find the intermediate 'anchor' with appropriate cache. Thus, the path obtained is optimal (i.e., closer to the SPT path) at the expense of large search costs. However, once

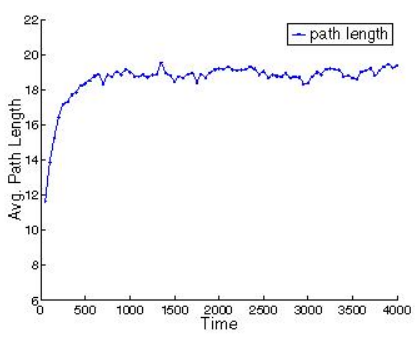

(a) Path Length

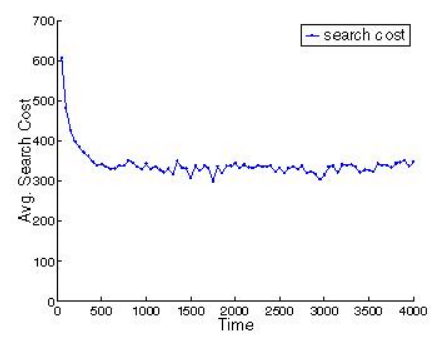

(b) Search Cost
Fig. 2. The Protocol Performance of FRESH Protocol vs. Time (RWK model, V $=5 \mathrm{~m} / \mathrm{s}$ ), For a Pair of Source and Destination.

the cache reaches the warm-cache phase, the FRESH protocol could utilize several intermediate 'anchors' to find the destination so that caching mechanism becomes a dominant factor. Hence, the path becomes less optimal but the search cost is significantly reduced.

\section{B. Expected Number of Encountered Nodes (Transi- tional Behavior)}

To better capture the transitional behavior, we measure how the expected number of encountered nodes (i.e., size of age gradient tree) increases with time. We observe that the transitional behavior of FRESH protocols is significantly affected by both mobility model and node velocity: For a given node velocity, the curves are different under different mobility patterns, as shown in Fig.3(a). Among them, the curve for RWK model is the lowest, the RWP and RPGM model are the highest ones, while the MH model lies in between. We also examine the effect of node velocity on the expected number of encountered nodes. As shown in Fig.3(b), in the RWP model, we observe that the expected number of encountered nodes increases faster when the node velocity becomes larger, which is intuitive. Similar results are also observed for RWK, RPGM and MH models.

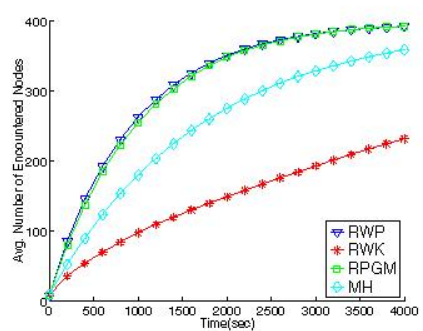

(a)Mobility Model ( $\mathrm{v}=10 \mathrm{~m} / \mathrm{s})$

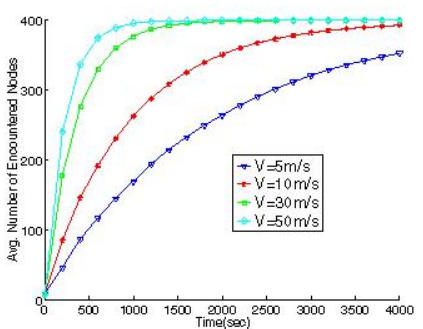

(b) Velocity (RWP model)
Fig. 3. The Impact of Mobility Model and Node Velocity on Average Number of Encountered Nodes vs. Time 


\section{Expected Path Length and Expected Search Cost (Steady Behavior)}

We also examine the detailed steady behavior, i.e., expected path length $\bar{P} L\left(x_{n}\right)$ and expected search cost $\overline{S C}\left(x_{n}\right)$ in FRESH for the destination with given SPT distance ( $n$ hops away from the source) $)^{3}$. Fig.4(a) and Fig.4(b) illustrate $\bar{P} L\left(x_{n}\right)$ and $\overline{S C}\left(x_{n}\right)$, under various mobility models at a given node velocity. The $\mathrm{x}$-axis in both figures is the SPT hop distance between a source and a destination, and the y-axis is the expected path length $\bar{P} L\left(x_{n}\right)$ or expected search cost $\overline{S C}\left(x_{n}\right)$ for the path with the given SPT distance, respectively. As shown in Fig.4(a), for a given SPT hop distance, the path length $\overline{P L}\left(x_{n}\right)$ for the $\mathrm{MH}$ model is the highest and the RWK model has the lowest value, while the values for RWP and RPGM models are nearly same and lie in between. As shown in Fig.4(b), $\overline{S C}\left(x_{n}\right)$ increases with the SPT hop distance $n$ for all the mobility patterns. For a given SPT hop distance, the RWP model generates the lowest overhead, and the other three mobility models are crossing over each other. When the hop distance $n$ is small, the MH model incurs a larger search cost than the RWK and RPGM model.

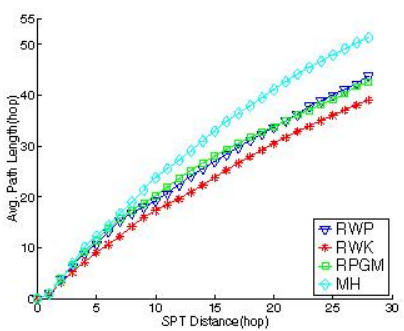

(a) Path Length

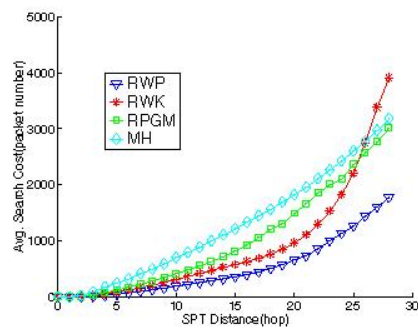

(b) Search Cost
Fig. 4. The Expected Path Length and Expected Search Cost obtained by FRESH protocol for all the Mobility Models $(\mathrm{v}=10 \mathrm{~m} / \mathrm{s})$

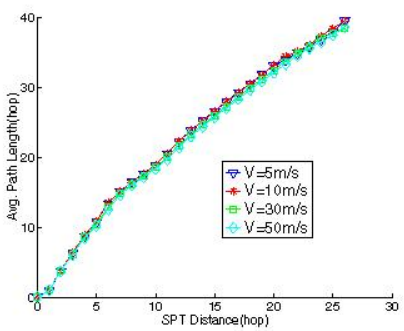

(a) Path Length

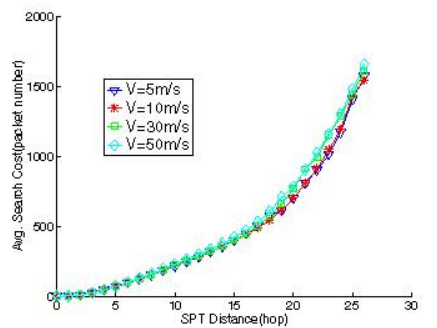

(b) Search Cost
Fig. 5. The Steady-State Protocol Performance under RWP Model, for Different Node Velocities.

${ }^{3}$ The SPT distance between node $\mathrm{A}$ and node $\mathrm{B}$, is the number of hops from node A to node B along the Shortest Path Tree rooted at node $\mathrm{A}$.
We also examine the effect of node velocity on the steady behavior of FRESH protocol for a given mobility model. We observed that the steady behavior of the FRESH protocol is insensitive to the node velocity for a given mobility pattern. Both $\overline{P L}\left(x_{n}\right)$ and $\overline{S C}\left(x_{n}\right)$ for RWP model are illustrated in the Fig.5(a) and Fig.5(b). Obviously, the different node velocity settings barely have an effect on the steady behavior of the FRESH protocol. Similar results are also observed for RWK, RPGM and MH models.

Intuitively, FRESH protocols are supposed to be highly sensitive to node mobility at both transitional state and steady state. However, partly contrary to our expectation, we find out that the FRESH protocol performance at transitional state is indeed sensitive to both mobility model and node velocity setting, while its steady state behavior is only sensitive to underlying mobility model but robust to node velocity. This further confirms the previous observation that the FRESH protocol behaves differently under RWP and RWK model [4], by using an even richer set of mobility models. Also, we shed some light on how the node velocity affects the FRESH protocol, which is a new addition to previous observation. In this paper, it is these observations that motivate us to develop the set of analytical models presented in Section VI and Section VII, in an attempt to explore the reasons behind them.

\section{Analytical Model for Node Encounters AND WARM-UP TIME}

We believe that the number of encountered nodes $E_{i}(t)$ is an important indicator to capture the cache warm-up process. Thus, we are particularly interested in developing analytical model to identify the relationship between the number of node encounters and its various impacting factors.

\section{A. Terminology}

We first define the terms in our analysis as follows.

1) $N$ : The number of mobile nodes in the network.

2) $A$ : The width of the square-shape network field.

3) $t$ : The time elapsed since the system starts, $0 \leq$ $t \leq T$, where $T$ is the overall system operational time.

4) $R$ : The communication range of wireless node.

5) $v$ : The average node velocity of mobile nodes.

6) $E_{i}(t)$ : The expected number of encountered nodes for node $i$ at time $t$, i.e., number of created (not updated) encounter table entries.

7) $\rho$ : The effective average node density in the field. 
8) $p_{i}(t)$ : The probability for node $\mathrm{i}$ that a node encountered at time $t$ is really a node that node $i$ never encountered in the history.

Note that if the mobile nodes are uniformly distributed over the simulation field, then, the node density is $\rho=$ $\frac{N}{A^{2}}$. In general, this is not valid for various mobility models and node distributions. Hence, in general, we assume that the node density is $\rho=\delta \frac{N}{A^{2}}$, where $\delta$ is the constant compensation factor for the non-uniformly fashion of node distribution(e.g., in $\mathrm{MH}$ model, where the nodes are only distributed along the freeway lanes, rather than uniformly distributed in the whole field). Different mobility models have different $\delta$ values.

Also, for random mobility model, for node $i$, the probability of encountering a new node (not encountered before) is $p_{i}(t)=\frac{N-E_{i}(t)}{N}$. But, in general, to consider all the mobility models, we assume that the probability of being the freshly encountered node is $p_{i}(t)=\kappa \frac{N-E_{i}(t)}{N}$, where $\kappa$ is the constant compensation factor for the nonuniform node encounter probability distribution(e.g., in RWK model, the newly encountered node is more likely to be a previously encountered node in contrast to RWP model, because the nodes in RWK model tend to wander in its own neighborhood due to the strong temporal correlation). Different mobility patterns have different $\kappa$ values.

\section{B. Expected Number of Encountered Nodes}

First, let us examine the number of nodes that node $i$ encountered during the time slot $\Delta t$ (after time $t$ ), i.e., $E_{i}(\Delta t \mid t)$. During that time slot, the node $i$ will travel in the distance $v \Delta t$, and the area covered by the wireless transmitter of node $i$ is $v \Delta t \times 2 R=2 R v \Delta t$ (Please note the "incremental" area covered during time interval $\Delta t$ (after time $t$ ) is not $2 R v \Delta t+\pi R^{2}$, since the area $\pi R^{2}$ had already been covered at time $t$ ). Because the node density in the field is $\rho$, the number of nodes that node $i$ encountered during time slot $\Delta t$ is $2 R v \Delta t \times \rho$. Within these encountered nodes, some are the nodes that node $i$ has never encountered in the past, while others are not. Thus, the number of 'freshly' encountered nodes for node $i$ during time slot $\Delta t$ (after time $t$ ) is

$$
\begin{aligned}
E_{i}(\Delta t \mid t) & =v \Delta t \times 2 R \times \rho \times p_{i}(t) \\
& =\delta \kappa(2 v R \Delta t) \frac{N}{A^{2}} \frac{N-E_{i}(t)}{N}
\end{aligned}
$$

The number of encountered nodes at time $t+\Delta t$ is sum of the number of encountered node at time $t$ and the number of 'freshly' encountered nodes in the time slot $\Delta t$. Hence,

$$
\begin{aligned}
E_{i}(t+\Delta t) & =E_{i}(t)+E_{i}(\Delta t \mid t) \\
& =\left(1-\delta \kappa \frac{2 v R}{A^{2}} \Delta t\right) E_{i}(t)+\delta \kappa \frac{2 v R N}{A^{2}} \Delta t
\end{aligned}
$$

Let $\alpha=\frac{2 v R}{A^{2}}, \beta=\frac{2 v R N}{A^{2}}$ and $\lambda=\delta \kappa$. Then, by using the above equation, we have

$$
\frac{E_{i}(t+\Delta t)-E_{i}(t)}{\Delta t}=\frac{d E_{i}(t)}{d t}=-\lambda \alpha E_{i}(t)+\lambda \beta
$$

Eqn.4 is a standard differential equation about the unknown function $E_{i}(t)$. Its general solution is given by

$$
E_{i}(t)=\frac{\beta}{\alpha}+c e^{-\lambda \alpha t}=N+c e^{-\lambda \frac{2 v R}{A^{2}} t}
$$

where $c$ is a constant factor to be determined.

When $t=0$, the nodes are static, and the encountered nodes are those within the radius range. Thus, the initial(boundary) condition is $E_{i}(0)=\lambda \pi N\left(\frac{R}{A}\right)^{2}$. Therefore, the constant factor $c=\left(\lambda \pi\left(\frac{R}{A}\right)^{2}-1\right) N$, and the number of encountered nodes over time is

$$
E_{i}(t)=N-N\left(1-\lambda \pi\left(\frac{R}{A}\right)^{2}\right) e^{-\lambda \frac{2 v R}{A^{2}} t}
$$

When the simulation time is long enough (i.e., approaches to infinity), the expected number of encountered node is $E_{i}(t \rightarrow \infty)=N$. At that time, any specific node has already encountered all the others.

Since the $\lambda$ values in Eqn. 6 are too complex to be analytically derived for various mobility models, we directly estimate this value from simulation via the maximum likelihood test, for different mobility models with the same given velocity $(\mathrm{v}=20 \mathrm{~m} / \mathrm{s})$. We observe that the $\lambda$ parameter is different for various mobility patterns. The $\lambda$ parameter for RWP, RWK, RPGM and MH models are $1.92325,0.71454,1.8324$ and 0.94365 , respectively (when $\mathrm{v}=20 \mathrm{~m} / \mathrm{s}$ ). We then applied the curving fitting scheme to compare the experiment results collected from simulations and the analytical results based on Eqn. 6 by using this set of $\lambda$ values, for different mobility models with all the velocity settings (except $\mathrm{v}=20 \mathrm{~m} / \mathrm{s}$ ). We compare the error margin ratio between the simulation results and results obtained from the analytical model. We find that the error margin ratio appears to be very small $(\leq 2 \%$ in most cases), across all the mobility models and all the velocity settings. 


\section{Warm-up Time}

The warm-up time $t_{\text {warmup }}$ is defined as the time when the node encounter ratio exceeds a portion (i.e., $\gamma)^{4}$ of all the other nodes. Hence, from Eqn.6, we are able to estimate the system warm-up time as

$$
t_{\text {warmup }} \geq \frac{\ln \left(\frac{1-\gamma}{\left(1-\lambda \pi\left(\frac{R}{A}\right)^{2}\right)}\right)}{-\lambda \frac{2 v R}{A^{2}}}
$$

From Eqn.7, we can see that the warm-up time is a function of several key system parameters: the node velocity $v$, radius range $R$, and constant compensation factor $\lambda$ which is unique for each mobility model.

\section{Analytical Models for Expected Path LENGTH AND EXPECTED SEARCh COST}

At the warm-cache phase, we are particularly interested in expected path length $\bar{P} L\left(x_{m}\right)$ and expected search cost $\overline{S C}\left(x_{m}\right)$. We identify that these two performance metrics of FRESH protocol are directly determined by the characteristics of age gradient tree.

\section{A. Terminology}

We first introduce the terms used in this section.

1) $x_{m}, x_{n}$ : The node $x_{m}\left(x_{n}\right)$ is the anchor node with $m$-hops ( $n$-hops) SPT distance from the destination. For each search step in the FRESH operation, the upstream anchor $x_{m}$ discovers its downstream anchor $x_{n}$ along the age gradient tree $(\forall n, \forall m, 0 \leq$ $m, n \leq D$ ). Please note $m$ is not always greater than $n$ in the FRESH protocol, even though $x_{m}$ is the upstream anchor.

2) $p\left(x_{m}, x_{n}\right)$ : The probability that upstream anchor $x_{m}$ discovers downstream anchor $x_{n}$.

3) $\bar{d}\left(x_{m}, x_{n}\right)$ : The average distance from upstream anchor $x_{m}$ to downstream anchor $x_{n}$, in terms of hop counts.

4) $\bar{c}\left(x_{m}, x_{n}\right)$ : The average search cost from upstream anchor $x_{m}$ to its downstream anchor $x_{n}$, in terms of transmitted packets.

5) $\overline{P L}\left(x_{m}\right)$ : The expected path length from anchor $x_{m}$ to the destination via the path computed by the FRESH protocol, in terms of hop count.

6) $\overline{S C}\left(x_{m}\right)$ : The expected search cost from anchor $x_{m}$ to the destination, generated by the FRESH protocol, in terms of transmitted packets.

\footnotetext{
${ }^{4}$ In our study, we define $\gamma$ as $30 \%$. As observed through simulations, both the characteristics of age gradient tree and the FRESH protocol performance become stable after $E(t)$ is larger than $30 \%$ of total node numbers.
}

7) $\mu_{n, m}$ : The ratio of the path length for $x_{n}$ to the path length for $x_{m}$, in the FRESH protocols, where $n>m$. In other words, $\mu_{n, m}=\frac{\bar{P} L\left(x_{n}\right)}{\bar{P} L\left(x_{m}\right)}$, (where $n>m)$.

8) $\nu_{n, m}$ : The ratio of the search cost for $x_{n}$ to the search cost for $x_{m}$, in the FRESH protocols, where $n>m$. In other words, $\nu_{n, m}=\frac{\overline{S C\left(x_{n}\right)}}{\overline{S C}\left(x_{m}\right)}$, (where $n>m$ ).

Here, note that the parameters $p\left(x_{m}, x_{n}\right), \bar{d}\left(x_{m}, x_{n}\right)$ and $\bar{c}\left(x_{m}, x_{n}\right)$ are the characteristics of the age gradient tree representing the probability, the expected distance and the expected cost from the upstream anchor to the downstream anchor, respectively.

\section{B. Expected Path Length and Expected Search Cost}

In the FRESH protocol, each upstream anchor $x_{m}$ is in charge of searching its downstream anchor $x_{n}$ along the age gradient tree. The average distance from the upstream anchor $x_{m}$ to the downstream anchor $x_{n}$ is $\bar{d}\left(x_{m}, x_{n}\right)$. Considering the expected path length for the anchor $x_{n}$ is given as $\bar{P} L\left(x_{n}\right)$, then, the path length from anchor $x_{m}$ to the destination via anchor $x_{n}$ is calculated as $\bar{P} L\left(x_{n}\right)+\bar{d}\left(x_{m}, x_{n}\right)$. Also, the probability that upstream anchor $x_{m}$ discovers its downstream anchor $x_{n}$ is given as $p\left(x_{m}, x_{n}\right)$. Hence,

$$
\begin{array}{r}
\overline{P L}\left(x_{m}\right)= \\
\sum_{n=1}^{D}\left(\bar{d}\left(x_{m}, x_{n}\right)+\bar{P} L\left(x_{n}\right)\right) p\left(x_{m}, x_{n}\right) \\
=\underbrace{\sum_{n=1}^{m-1}\left(\bar{d}\left(x_{m}, x_{n}\right)+\bar{P} L\left(x_{n}\right)\right) p\left(x_{m}, x_{n}\right)}_{(1)} \\
+\underbrace{\left(\bar{d}\left(x_{m}, x_{m}\right)+\bar{P} L\left(x_{m}\right)\right) p\left(x_{m}, x_{m}\right)}_{(2)} \\
+\underbrace{\sum_{n=m+1}^{D}\left(\bar{d}\left(x_{m}, x_{n}\right)+\overline{P L}\left(x_{n}\right)\right) p\left(x_{m}, x_{n}\right)}_{(3)}
\end{array}
$$

where $\mathrm{D}$ is network diameter. Here, part(1), part(2) and part(3) corresponds to the cases in which the SPT distance from upstream anchor to the destination ( $m$ hops) is larger than, equal to, or smaller than the SPT distance from the downstream anchor to the destination ( $n$ hops), respectively. The equation could be solved in a recursive manner. For the upstream anchor $x_{m}$ with a given hop distance $m$, the path length for the downstream anchor $\bar{P} L\left(x_{n}\right)(\forall n, n<m)$ in part(1) is already known. For the case where $n>m$ (in part (3)), by substituting $\bar{P} L\left(x_{n}\right)=\mu_{n, m} \bar{P} L\left(x_{m}\right)$ (where $n>m$ ) into part(3) of 
Eqn.9 and then simplifying Eqn.9, we get the expected path length for anchor $x_{m}$ along the path obtained by FRESH protocol as

$$
\overline{P L}\left(x_{m}\right)=\frac{\sum_{n=1}^{D} \bar{d}\left(x_{m}, x_{n}\right) p\left(x_{m}, x_{n}\right)+\sum_{n=1}^{m-1} \overline{P L}\left(x_{n}\right) p\left(x_{m}, x_{n}\right)}{1-p\left(x_{m}, x_{m}\right)-\sum_{n=m+1}^{D} \mu_{n, m} p\left(x_{m}, x_{n}\right)}
$$

Similarly, we get the expected search cost as

$$
\overline{S C}\left(x_{m}\right)=\frac{\sum_{n=1}^{D} \bar{c}\left(x_{m}, x_{n}\right) p\left(x_{m}, x_{n}\right)+\sum_{n=1}^{m-1} \overline{S C}\left(x_{n}\right) p\left(x_{m}, x_{n}\right)}{1-p\left(x_{m}, x_{m}\right)-\sum_{n=m+1}^{D} \nu_{n, m} p\left(x_{m}, x_{n}\right)}
$$

However, the parameters $\mu_{n, m}$ in Eqn.10 and $\nu_{n, m}$ in Eqn.11 remain unknown. Through our derivations, we find that the path length of FRESH paths is linearly correlated with its SPT path length. Hence, the parameter $\mu_{n, m}$ is

$$
\mu_{n, m}=\frac{\bar{P} L\left(x_{n}^{F R E S H}\right)}{\bar{P} L\left(x_{m}^{F R E S H}\right)} \approx \frac{n}{m}
$$

Also, we find that search cost of FRESH path is polynomially correlated with its SPT path length. To be in details, the parameter $\nu_{n, m}$ is also a function of their SPT distances, as

$$
\nu_{n, m}=\frac{\overline{S C}\left(x_{n}^{F R E S H}\right)}{\overline{S C}\left(x_{m}^{F R E S H}\right)} \approx \frac{2 n^{3}-3 n^{2}+n}{2 m^{3}-3 m^{2}+m}
$$

We also validate both Eqn.12 and Eqn.13 by taking the measurement of the path length ratio $\mu_{n, m}$ and search cost ratio $\nu_{n, m}$ (where $n>m$ ) in the FRESH protocol, through simulations. The results indicate that, in most cases, Eqn.12 and Eqn.13 are reasonable approximations of real scenarios ${ }^{5}$.

\footnotetext{
${ }^{5}$ We acknowledge that the both equations (Eqn.12 and Eqn.13) are only the approximations of the realistic scenarios. However, the accuracy of the analytical models (Eqn.10 and Eqn.11) will not be significantly affected. This is because, intuitively, we know that it is a rare case that an upstream anchor will search a downstream anchor whose SPT distance to destination is even much larger than its own SPT distance to the destination. Through the simulation, we observe that $p\left(x_{m}, x_{n}\right)$ is a very small value if $m \leq n \leq$ $m+\varepsilon$, and $p\left(x_{m}, x_{n}\right)=0$ if $n \geq m+\varepsilon$ (in most mobility scenarios, $\varepsilon \leq 2$ ). At the same time, the value $p\left(x_{m}, x_{n}\right)$ (where $n<m$ ) seems to be a very large value compare to the value $p\left(x_{m}, x_{n}\right)$ if $n>m$. That is to say, even the approximation of $\mu_{n, m}$ and $\nu_{n, m}$ is not exactly accurate, the small values of $p\left(x_{m}, x_{n}\right)$ $(n>m)$ enables the item $\sum_{n=m+1}^{D} \mu_{n, m} p\left(x_{m}, x_{n}\right)$ (in Eqn.10) and $\sum_{n=m+1}^{D} \nu_{n, m} p\left(x_{m}, x_{n}\right)$ (in Eqn.11) to be very small values. Thus, the accuracy of the estimation based on Eqn.15 and Eqn.16 will not be affected significantly by these approximations made in Eqn.12 and Eqn.13. We further validate our argument through extensive simulations.
}

After replacing the $\mu_{n, m}$ factor and $\nu_{n, m}$ factor by Eqn.12 and Eqn.13, we get the analytical models for the expected path length and the expected search cost in the FRESH protocol (in recursive format) as

$$
\begin{gathered}
\overline{P L}\left(x_{m}\right)=\frac{\sum_{n=1}^{D} \bar{d}\left(x_{m}, x_{n}\right) p\left(x_{m}, x_{n}\right)+\sum_{n=1}^{m-1} \bar{P} L\left(x_{n}\right) p\left(x_{m}, x_{n}\right)}{1-p\left(x_{m}, x_{m}\right)-\sum_{n=m+1}^{D} \frac{n}{m} p\left(x_{m}, x_{n}\right)} \\
\text { withP } L\left(x_{1}\right)=1
\end{gathered}
$$

As shown in Eqn.14, for a given $m$ value, the expected path length $\bar{P} L\left(x_{m}\right)$ is a function of the characteristics of age gradient tree $\left(\bar{d}\left(x_{m}, x_{n}\right), p\left(x_{m}, x_{n}\right)\right)$ and the expected path length of the paths whose SPT distance smaller than $m$ hops $\left(\bar{P} L\left(x_{n}\right)\right.$, where $\left.1 \leq n<m\right)$. Similarly, as shown in Eqn.15, the expected search cost $\overline{S C}\left(x_{m}\right)$ is also a function of the characteristics of age gradient tree $\left(\bar{c}\left(x_{m}, x_{n}\right), p\left(x_{m}, x_{n}\right)\right)$ and the expected search cost of the paths whose SPT distance is smaller than $m$ hops $\left(\overline{S C}\left(x_{n}\right)\right.$, where $\left.1 \leq n<m\right)$.

Both Eqn.14 and Eqn.15 should be solved in a recursive fashion, from smallest $m$ value $(m=1)$ to the largest $m$ value $(m=D)$. Intuitively, the initial conditions for recursive-format Eqn. 14 and Eqn. 15 are $\bar{P} L\left(x_{1}\right)=1$ and $\overline{S C}\left(x_{1}\right)=1$. Starting from these initial conditions, we are able to estimate the expected path length $\bar{P} L\left(x_{m}\right)$ and expected search cost $\overline{S C}\left(x_{m}\right)$ based on these two analytical models, if the characteristics of age gradient tree $\left(\bar{d}\left(x_{m}, x_{n}\right), \bar{c}\left(x_{m}, x_{n}\right)\right.$ and $\left.p\left(x_{m}, x_{n}\right)\right)$ are given.

Again, we compute the error margin ratio between the calculated result (based on Eqn.14 and Eqn.15) and the measured result (from simulation). Through the study, we find the error margin ratio for the expected path length is $5 \%-10 \%$ and the error margin ratio for the expected search cost is around $6 \%-17 \%$ in all the mobility scenarios. The acceptable error margin ratios indicates that the proposed analytical models are good approximations to study the steady behavior of FRESH protocol based on the measurement of the characteristics of age gradient tree $\left(\bar{d}\left(x_{m}, x_{n}\right), \bar{c}\left(x_{m}, x_{n}\right)\right.$ and $\left.p\left(x_{m}, x_{n}\right)\right)$.

\section{THE LOGICAL RELATIONSHIP BETWEEN Mobility, Age Gradient Tree And FRESH PROTOCOL}

Finally, we attempt to answer questions Why FRESH protocol is, or, is not, affected by the underlying mobility 
scenarios?, by developing a logical relationship among all the components.

\section{A. Temporal-Spatio Correlation and Age Gradient Tree}

Temporal-spatio correlation, which exists for all the mobility models discussed in this paper, can be used to roughly estimate the distance between nodes, based on their last encounter time. However, note that different mobility models exhibit different types of temporalspatio correlation. To vividly illustrate the temporalspatio correlation, we examine the 3-D age gradient field. For a specific destination, the age gradient field is formed if each node on the 2-D space is associated with its encounter age for the destination (as its z-axis), which represents the 'potential' (similar to the meaning of 'potential' in physics). As shown in Fig.6(a) and Fig.6(b), the age gradient fields for RWK model and MH model are like funnels (whose sink is the given destination), indicating the spatial distance between a node and a destination is somehow correlated with their encounter age. Similar temporal-spatio correlation is also clearly observed for RWP and RPGM models.

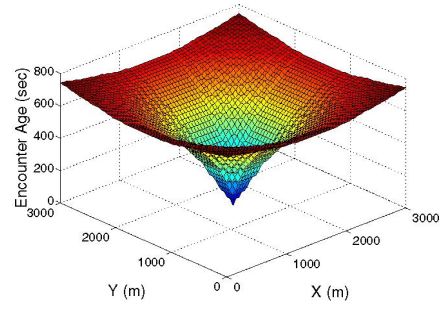

(a) RWK model

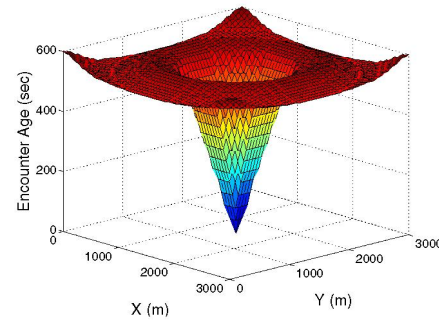

(b) $\mathrm{MH}$ model
Fig. 6. The Temporal-Spatio Correlation of a given destination for the RWK and MH model (V=30m/s), measured from simulation. We pick one node close to the center of simulation field as the destination, for better illustration.

The age gradient field implicitly designates the route from any node to the given destination. In other words, because of this temporal-spatio correlation, the packets are able to gradually move from upstream anchor towards downstream anchor and finally reach the destination. Hence, we believe that temporal-spatio correlation is the key reason enabling the mobility-assisted encounter-based FRESH protocol. FRESH protocol will not function well if this temporal-spatio correlation does not exist.

Rather than being used directly, the 3-D temporalspatio correlation and the designated routes on the funnel surface are projected onto the 2-D space, forming an age gradient tree (exactly the same 'age gradient tree'

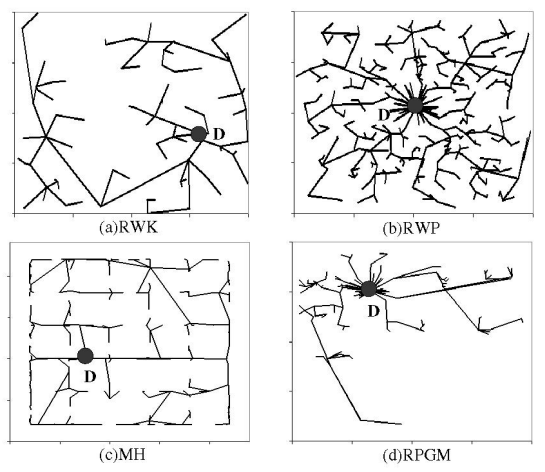

Fig. 7. The Age Gradient Tree (AGT) for RWP, RWK, MH and RPGM models $(v=20 \mathrm{~m} / \mathrm{s}, \mathrm{t}=400 \mathrm{sec})$. Here, $\mathrm{D}$ is the destination, and also, the root of AGT.

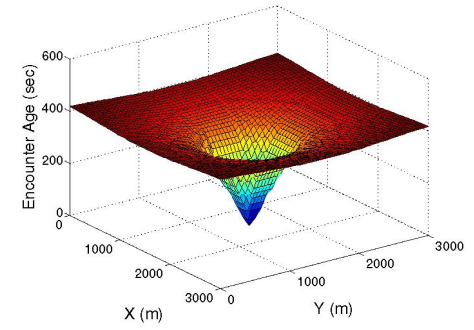

Fig. 8. The Temporal-Spatio Correlation of a given destination near center, for the RWK model $(\mathrm{V}=50 \mathrm{~m} / \mathrm{s})$, measured from simulation.

in Section III-B). Therefore, age gradient tree is an abstracted form of the temporal-spatio correlation.

\section{B. The Impact of Mobility on Age Gradient Tree and FRESH Protocol Performance}

Through Section V, we know that the performance of FRESH protocol at steady state is fully determined by characteristics of age gradient tree. Here, we mainly focus on the impact of mobility on the behavior of age gradient tree at steady state, i.e., how the shape and the characteristics of age gradient tree behave under different mobility models and node velocities. First, we discuss the case of different mobility models with same velocity. Intuitively, different mobility models create different encounter patterns and hence the different types of temporal-spatio correlation, as shown in Fig.6(a) (RWK) and Fig.6(b) (MH). Also, as shown in Fig.7, the shape of age gradient tree for RWK, RWP, RPGM and MH mobility models are visually different (and so are their characteristics). For example, in the $\mathrm{MH}$ model, the age gradient tree consists of age gradients on the horizontal and vertical lines because nodes are restricted to the Manhattan map (as shown in Fig.7(c)). Next, we examine the case of same mobility model with different velocities. Here, interestingly, we observe that the shapes and characteristics of age gradient tree for the 
same mobility model are similar under the different node velocities. Quantitatively, for a given mobility model, the error margin ratio for $p\left(x_{m}, x_{n}\right), \bar{d}\left(x_{m}, x_{n}\right)$ and $\bar{c}\left(x_{m}, x_{n}\right)$ between different velocities is very small (less than $5.25 \%, 4.06 \%$ and $8.45 \%$, for all these four mobility models). To sum up, for steady state, we observe that the mobility model significantly affects the shape and the characteristics of the age gradient tree, while the velocity settings may not.

This counter-intuitive observation can be explained in this way: With the different node velocity settings, the exact encounter age between nodes may differ and the height of 3-D temporal-spatio correlation may be different (e.g., the height of funnel is around $800 \mathrm{sec}$ in Fig.6(a), while the height is about $400 \mathrm{sec}$ in Fig.8). However, its rough shape and the designated routes on the funnel surface (the age gradient from the node with larger encounter age to the node with smaller encounter age) do not change drastically, as shown in Fig.6(a) $(v=30 \mathrm{~m} / \mathrm{s})$ and Fig. $8(v=50 \mathrm{~m} / \mathrm{s})$. In other words, once the 3-D temporal-spatio correlation is projected to the 2-D field, the information of exact encounter age (z-axis value) becomes useless. In this way, the node velocity only contributes to 'scale' the encounter age but does not affect their relative relationship which forms the age gradient tree. Hence, the shapes (and characteristics) of age gradient tree under different node velocity are nearly same for a given mobility model.

Therefore, the FRESH protocol, whose performance is determined by the characteristics of the age gradient tree, behaves differently for various mobility models but less sensitive to different node velocities. We, thus, believe that the characteristics of age gradient tree are the key bridge linking the mobility effect and the protocol behavior of FRESH protocol ${ }^{6}$.

\section{COnclusion \& Future Work}

In this paper, we quantitatively analyze the complicated interaction between the mobility and the last encounter based protocol (i.e., FRESH) which directly utilize mobility information to discover node location or route. First, we evaluated the FRESH protocol over a rich set of mobility models by which we believe that mobility space could be spanned. We found that the transitional behavior of FRESH protocol is significantly affected by

\footnotetext{
${ }^{6}$ The role of age gradient tree in FRESH protocol is same to that of Link Duration and Path Duration in the SPT-based MANET routing protocols [9]. We believe that, because of the different protocol mechanisms, it is necessary to examine the different connectivity graph properties which are directly relevant to the protocol mechanism.
}

underlying mobility scenarios, while its steady behavior is less sensitive to the node mobility. Motivated by these observations, we developed a set of analytical models to capture the FRESH protocol behavior at both transitional state and steady state, and we also realize that age gradient tree is the key to explain the complex interplay between node mobility and last encounter based protocol.

The analytical models and qualitative analysis not only deepen our theoretical understanding itself, but also contribute to practical protocol design enhancement. Partly inspired by the fact that the age gradient tree is insensitive to the node velocity, we also aim to design a more practical protocol whose performance is robust to node velocity(based on Eqn.15 and Eqn.16). Through our study, we feel that AGT-based mobility-assisted protocols hold great potentials for the future wireless network, and this topic deserves more attention from research society.

\section{REFERENCES}

[1] K. Fall. "A delay-tolerant network architecture for challenged internets", In Proceeding of ACM SIGCOMM, 2003.

[2] M. Grossglauser and D. Tse. "Mobility increases the capacity of ad-hoc wireless networks", In: Proceeding of IEEE INFOCOM 2001.

[3] T. Small and Z. Haas. "The Shared Wireless Infostation Model - A New Ad Hoc Networking Paradigm (or Where there is a Whale, there is a Way)". In Proceeding of ACM MobiHoc, June 2003.

[4] H. Dubois-Ferriere, M. Grossglauser, and M. Vetterli. "Age matters: Efficient route discovery in mobile ad hoc networks using encounter ages", In Proceeding of ACM MobiHoc, June 2003

[5] M. Grossglauser and M. Vetterli. "Locating nodes with EASE: Mobility diffusion of last encounters in ad hoc networks", In Proceeding of IEEE INFOCOM, April 2003.

[6] J. Broch, D.A. Maltz, D.B. Johnson, Y.-C. Hu, J. Jetcheva, "A performance comparison of multi-hop wireless ad hoc network routing protocols", in: Proceedings of ACM Mobicom 1998, Roma, Italy.

[7] F. Bai, N. Sadagopan, A. Helmy, "IMPORTANT: a framework to systematically analyze the impact of mobility on performance of routing protocols for ad hoc networks", in INFOCOM 2003.

[8] W. Zhao, M. Ammar and E. Zegura, "A Message Ferrying Approach for Data Delivery in Sparse Mobile Ad Hoc Networks". In Proceedings of ACM MobiHoc 2004, Tokyo Japan.

[9] F. Bai, N. Sadagopan, B. Krishnamachari, A. Helmy, "Modeling Path Duration Distributions in MANETs and their Impact on Routing Performance", IEEE Journal on Selected Areas of Communications (JSAC), Vol. 22, No. 7, pp. 13571373, September 2004. 